\title{
Biomarkers and genetic modulators of cerebral vasculopathy in sub-Saharan ancestry children with sickle cell anemia
}

\author{
Marisa Silva $^{\mathrm{a}}$, Sofia Vargas ${ }^{\mathrm{a}}$, Andreia Coelho ${ }^{\mathrm{a}}$, Emanuel Ferreira ${ }^{\mathrm{a}}$, Joana Mendonça ${ }^{\mathrm{a}}$, \\ Luís Vieira $^{\mathrm{a}, \mathrm{b}}$, Raquel Maia ${ }^{\mathrm{c}}$, Alexandra Dias ${ }^{\mathrm{d}}$, Teresa Ferreira ${ }^{\mathrm{d}}$, Anabela Morais ${ }^{\mathrm{e}, 1}$, \\ Isabel Mota Soares ${ }^{\mathrm{f}}$, João Lavinha ${ }^{\mathrm{a}, \mathrm{g}}$, Rita Silva ${ }^{\mathrm{h}}$, Paula Kjöllerström ${ }^{\mathrm{c}}$, Paula Faustino ${ }^{\mathrm{a}, \mathrm{i}, *}$ \\ ${ }^{a}$ Departamento de Genética Humana, Instituto Nacional de Saúde Dr. Ricardo Jorge, Lisbon, Portugal \\ ${ }^{\mathrm{b}}$ ToxOmics, Faculdade de Ciências Médicas, Universidade Nova de Lisboa, Lisboa, Portugal \\ ${ }^{\mathrm{c}}$ Unidade de Hematologia, Hospital de Dona Estefânia, Centro Hospitalar Universitário de Lisboa Central (CHULC), Lisbon, Portugal \\ ${ }^{\mathrm{d}}$ Núcleo de Hematologia, Departamento de Pediatria, Hospital Prof. Doutor Fernando Fonseca, Amadora, Portugal \\ ${ }^{\mathrm{e}}$ Departamento de Pediatria, Hospital de Santa Maria, Centro Hospitalar Universitário de Lisboa Norte, Lisbon, Portugal \\ ${ }^{\mathrm{f}}$ Departamento de Pediatria, Hospital Garcia de Orta, Almada, Portugal \\ ${ }^{\mathrm{g}}$ BioISI, Faculdade de Ciências, Universidade de Lisboa, Lisbon, Portugal \\ ${ }^{\text {h }}$ Unidade de Neuropediatria, Hospital de Dona Estefânia, CHULC, Lisbon, Portugal \\ ${ }^{\mathrm{i}}$ Instituto de Saúde Ambiental (ISAMB), Faculdade de Medicina, Universidade de Lisboa, Lisbon, Portugal
}

\section{A R T I C L E I N F O}

Editor: Mohandas Narla

Keywords:

Sickle cell anemia

Cerebral vasculopathy

Ischemic stroke

Genetic modulators

Biomarkers

\begin{abstract}
A B S T R A C T
We investigated biomarkers and genetic modulators of the cerebral vasculopathy (CV) subphenotype in pediatric sickle cell anemia (SCA) patients of sub-Saharan African ancestry. We found that one VCAM1 promoter haplotype (haplotype 7) and VCAM1 single nucleotide variant rs1409419_T were associated with stroke events, stroke risk, as measured by time-averaged mean of maximum velocity in the middle cerebral artery, and with high serum levels of the hemolysis biomarker lactate dehydrogenase. Furthermore, VCAM-1 ligand coding gene ITGA4 variants rs113276800_A and rs3770138_T showed a positive association with stroke events. An additional positive relationship between a genetic variant and stroke risk was observed for ENPP1 rs1044498_A. Conversely, NOS3 variants were negatively associated with silent cerebral infarct events (VNTR 4b_allele and haplotype V) and CV globally (haplotype VII). The -alpha ${ }^{3.7 \mathrm{~kb}}$-thal deletion did not show association with CV. However, it was associated with higher red blood cell and neutrophil counts, and lower mean corpuscular volume, mean corpuscular hemoglobin and red cell distribution width.

Our results underline the importance of genetic modulators of the CV sub-phenotype and their potential as SCA therapeutic targets. We also propose that a biomarker panel comprising biochemical, hematological, imaging and genetic data would be instrumental for CV prediction, and prevention.
\end{abstract}

\section{Introduction}

Sickle cell anemia (SCA), the homozygous form of the c:20A $>\mathrm{T}$ mutation in the beta-globin gene, is the most common and severe presentation of sickle cell disease (SCD). High birth rates and child mortality are most frequent in sub-Saharan Africa, however, population movements are leading to a wider distribution, emphasizing the global health emergence status of the disease [1-3]. In children, the most common sub-phenotypes are cerebral vasculopathy (CV), acute chest syndrome, hyposplenism, renal disease and painful crises. CV is a major complication and comprises overt stroke, silent cerebral infarcts (SCIs), transient ischemic attacks and frequently neurocognitive complications

\footnotetext{
Abbreviations: SCA, sickle cell anemia; SCD, sickle cell disease; CV, cerebral vasculopathy; SCI, silent cerebral infarction(s); LDH, lactate dehydrogenase; TAMMV,

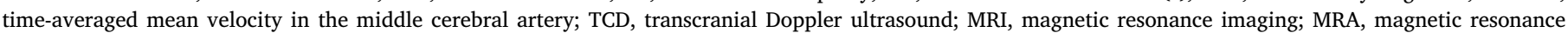

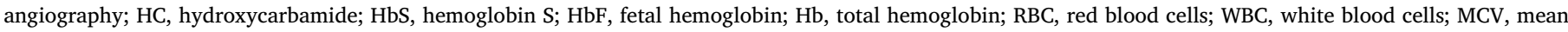

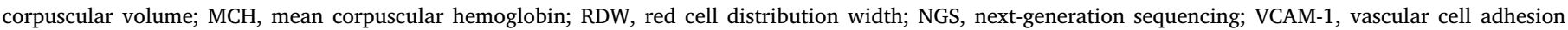
molecule 1; ITGA4, very-late antigen 4; eNOS, endothelial nitric oxide synthase; NO, nitric oxide; TFBS, transcription factor binding site(s)

* Corresponding author at: Departamento de Genética Humana, Instituto Nacional de Saúde Dr. Ricardo Jorge, Avenida Padre Cruz, $1649-016$ Lisboa, Portugal.

E-mail address: paula.faustino@insa.min-saude.pt (P. Faustino).

${ }^{1}$ Deceased co-author.
} 
at a later stage. Children with SCA have a much higher risk of stroke than the general pediatric population. The prevalence of overt stroke approaches $11 \%$ by age 20 years. On the other hand, SCIs have been found in up to $37 \%$ of children with SCA [4].

The current standard of care for stroke prevention in SCA children is Transcranial Doppler (TCD) screening - through measurement of timeaveraged mean velocity (TAMMV) in the middle cerebral artery - followed by regular blood transfusions therapy and hydroxycarbamide (HC) treatment. Despite its high sensitivity, TCD still does not allow identification of all SCA children that will experience a stroke and, conversely, children with high TAMMV ( $>200 \mathrm{~cm} / \mathrm{s}$ ) may not develop stroke [5]. Moreover, blood transfusion/HC therapies are not without limitations or adverse side effects [6]. On the other hand, although diagnosis with magnetic resonance imaging, MRI, (or angiography, MRA) is recommended for early diagnosis of SCIs and recognition of large vessel stenosis, MRI/MRA are not useful to identify patients at risk of developing SCIs or large vessel stenosis [7]. A more specific and sensitive panel of biomarkers for CV prediction and prognosis, that includes genetic variants with disease modifying effects, is therefore of the utmost importance.

In previous studies, we identified variants in VCAM1, NOS3 and $H B A$ with a positive association with chronic hemolysis, a known pathophysiological SCA mechanism [8]. Building on those results we aimed, in this work, to assess if those variants were also associated with pediatric CV in a sub-Saharan SCA population. Our candidate gene approach also focused on the VCAM-1 ligand gene, ITGA4, and for comparison purposes, we included the three genetic variants (PON1 rs662, ENPP1 rs1044498 and GOLGB1 rs3732410) previously reported in association with pediatric stroke in SCA patients $[7,9,10]$.

\section{Materials and methods}

\subsection{Ethical statement}

Ethical approval for the study was granted by the institutional review boards of Instituto Nacional de Saúde Dr. Ricardo Jorge (INSA) and of participant hospitals, in line with the principles of the Declaration of Helsinki. The aim and study procedures were explained to the children's parents (or legal guardians) and they provided informed written consent prior to their enrolment in this study.

\subsection{Study population}

This case-control study was performed at INSA in cooperation with four hospitals in the Greater Lisbon area - Hospital D. Estefânia, Hospital de Santa Maria, Hospital Fernando Fonseca and Hospital Garcia de Orta, the four largest centers of that metropolitan area. These centers combined receive the highest numbers of SCA pediatric patients in our country. Seventy unrelated pediatric patients ( $\geq 3$ years old) of direct sub-Saharan African ancestry diagnosed with SCA were selected. Exclusion criteria included age $<3$ years old, non-African ancestry, previous HC treatment or having received a blood transfusion in the 120 days prior to enrolment.

Data obtained from patients and parents (or legal guardians) interviews, which included demographic characteristics (age, gender, parents' geographic origin), were collected. Hemoglobin profiles (HbS, $\mathrm{HbF}$ ), hematological parameters [RBC, leukocyte, neutrophil and platelet counts, mean corpuscular volume (MCV), mean corpuscular hemoglobin (MCH), red cell distribution width (RDW)] as well as hemolysis biochemical and hematological biomarkers [serum LDH and total bilirubin and reticulocyte count] were retrospectively collected from patients' hospital records. All these parameters were obtained by standard procedures and HbF levels, in particular, were measured by highperformance liquid chromatography (HPLC). The data collected for each parameter were the result of, at least, three different time-point measurements, performed in steady-state periods, and prior to any treatment with HC and more than $>120$ days after receiving a blood transfusion. The patients were analyzed according to CV outcome, or stroke risk, in the following groups (i) overt ischemic stroke ( $\geq 1$ episode), henceforth designated as "stroke" $(n=62)$; (ii) "silent cerebral infarct" ( $\geq 1$ event), SCI $(n=53)$; (iii) stroke and/or SCI, as confirmed by MRI or MRA, $(n=62)$; or "stroke risk" $(n=60)$, with risk stratification provided by the TAMMV values, obtained during TCD, as follows: (a) high risk - TAMMV $\geq 200 \mathrm{~cm} / \mathrm{s}$; (b) conditional (or moderate) risk $-200 \mathrm{~cm} / \mathrm{s}>$ TAMMV $\geq 170 \mathrm{~cm} / \mathrm{s}$; and (c) low risk TAMMV $<170 \mathrm{~cm} / \mathrm{s}$.

\subsection{Genotyping}

Genomic DNA was isolated from peripheral blood samples of each patient using the MagNA Pure LC Instrument (Roche Diagnostics $\mathrm{GmbH}$, Mannheim, Germany). All samples were anonymized and specific genotypes could be linked to phenotypes only through the main study database.

The homozygous status for the SCA mutation in the $H B B$ gene (c.20A > T) was confirmed by polymerase chain reaction followed by restriction fragment length analysis (PCR-RFLP) with the endonuclease Bsu36 I. Beta-globin cluster haplotypes were assigned after examining six restriction endonucleases sites within the cluster: $X m n$ I $\left(5^{\prime}\right.$ to $H B G 2$ ), Hind III (within the HBG2 and HBG1) Hinc II (within and $3^{\prime}$ to $\psi H B B$ ) and Hinf I ( $3^{\prime}$ to $H B B$ ). Aliquots of the amplified products were digested with the appropriate enzymes under the conditions recommended by the manufacturers. Haplotypes were determined by the presence or absence of cleavage at each site and by analyzing the compiled pattern through comparison to known patterns [11]. The $-\alpha^{3.7 \mathrm{~kb}}$-thal deletion was assessed by gap-PCR [12].

Putative modifier genes were identified through previous studies by our group [8] and from other published reports based on the influence on the phenotypes of interest. These candidate genes were used to identify and genotype SNPs and other variants in patient samples. For VCAM1, NOS3, PON1, ENPP1 and GOLGB1 genes genotyping was performed using TaqMan-based PCR with commercially available or customized primers.

\subsubsection{Screening for ITGA4 variants by next-generation sequencing (NGS)}

In order to search for variants in the regulatory region of ITGA4 gene, NGS analysis was used on a long PCR fragment (4.1 kb), including its flanking regions. PCR was performed using the primers FW5'-CAG AGGCTCATTAGGACCC-3' and Rv5'-CCTTGCGGTACTATCCAGGC-3' and the FailSafe enzyme with the PreMix G (Epicentre, Illumina, San Diego).

The NGS workflow consisted in five steps: PCR product purification using paramagnetic beads (Agentcourt, Ampure XP); double-stranded DNA quantification in a Qubit fluorometer; DNA library preparation using the Nextera XT kit (Illumina, San Diego) following the manufacturer's instructions; high throughput sequencing in a MiSeq benchtop sequencer (Illumina, San Diego); data analyses were performed using the following tools: Sequencing Analysis Viewer (v1.8.46, Illumina) and FastQC (v0.11.5, Babraham Bioinformatics, https://www. bioinformatics.babraham.ac.uk/projects/fastqc/) were used for quality analysis. The MiSeq ${ }^{\circledR}$ Reporter software package (v2.6.2, Illumina) was used for read mapping (with Burrows-Wheeler Aligner) and variant calling and filtering (with Genome Analysis Toolkit). FastQ screen (v.0.9.3, Babraham Bioinformatics) was used to screen for contamination between samples. Variant Effect Predictor (www.ensembl. org) was used to annotate variants and Integrative Genomics Viewer (v.2.3.86) (Broad Institute; [13]) was used for visualization of reads and variants. Validation of the variants was performed using automated Sanger sequencing, after amplification with customized primers in the 3130XI Genetic Analyser, (Applied Biosystems). The results were analyzed using FinchTV v.1.4.0 software (Geospiza, Inc.). The genotyping results were added to the previously created database. 


\subsubsection{Haplotype reconstruction}

Haplotype reconstruction was performed using PHASE software v2.1 developed by Mathew Stephens at Washington University, according to the developer's instructions (https://els.comotion.uw.edu/ express_license_technologies/phase). Haplotypes were reconstructed for genetic variants in NOS3 (rs2070744, intron 4_27 bp VNTR, rs1799983), in the promoter of VCAM1 (rs1409419, rs3917024, rs3917025, rs3783598, rs1041163, rs3783599) and for genetic variants of ITGA4 (rs1375493, rs35723031, rs10562650; rs1839269 and rs1839268).

\subsubsection{In silico analysis}

Population allele and genotype frequencies were recorded for each observed variant using the Ensembl browser (www.ensembl.org). SNPs' sequences were retrieved using the NCBI SNP search engine (http:// ncbi.nlm.gov/snp).

Transcription factor binding sites (TFBS) analysis was performed for the variants identified in the regulatory regions, using the MatInspector tool (Genomatix, Munich, Germany), to evaluate potential effects on the regulation of gene expression by altering putative TFBS. Only results above the 0.85 threshold were considered, which corresponds to a maximum of $15 \%$ dissimilarity between the identified sequence and the consensus sequence. A comparison with previously reported consensus sequences of TFBS for the VCAM1 [14] and the ITGA4 [15] promoters was performed. The sequences of the identified variants were not found to overlap with any of the previously reported TFBS consensus sequences. Hence, no strong effects for these genes' expression are to be expected as a result of the presence of those variants.

\subsubsection{Statistical analysis}

The analyses were performed using the SPSS software (version 25.0, IBM Inc., Chicago, USA). For descriptive analysis, continuous variables were represented as medians and interquartile ranges (IQR). To evaluate the Gaussian distribution of variables, Shapiro-Wilk normality tests where applied. We used the Mann-Whitney $U$ test to compare the medians of variables. Categorical variables are represented as number, frequencies and percentages. The chi-square test or the Fisher's exact test were used to compare categorical variables on bivariate analysis. Statistical significance was defined as $p<0.05$.

The minor allele for each variant was evaluated for potential association with stroke, SCI, cerebral vasculopathy (stroke and SCI combined) or risk (as measured by TCD-TAMMV values), via $2 \times 2$ phenotype $\times$ genotype contingency tables. Only polymorphisms with a minor allele frequency (MAF) $>5 \%$ were considered for association analysis. Phenotypes were classified as follows: (i) "stroke" (at least one previous overt ischemic stroke event, as confirmed by MRI/MRA) or "no stroke" (no clinically/imaging identified stroke event); (ii) "SCI" (at least one event as identified by MRI/MRA) or "no SCI" (no SCI events, as confirmed by MRI); (iii) "cerebral vasculopathy" (at least one overt ischemic stroke and/or SCI event) or "no cerebral vasculopathy"; and (iv) "risk of stroke" (high/moderate: TAMMV $\geq 200 \mathrm{~cm} / \mathrm{s}$ or $199 \geq$ TAMMV $\geq 170 \mathrm{~cm} / \mathrm{s}$; low: TAMMV $<170 \mathrm{~cm} / \mathrm{s}$ ).

Each variant was also evaluated for potential association with biochemical and hematological parameters, including hemolysis biomarkers (LDH, total bilirubin, reticulocyte count).

\section{Results}

\subsection{Population description and genotyping}

This study was performed on seventy unrelated SCA patients, (age range: $3-16$ years, 40 males, 30 females), living in Portugal but of direct sub-Saharan ancestry, with parental geographic origin mainly from Angola, São Tomé and Príncipe and Cape Verde (Table 1).
Table 1

Demographic, neurological status and laboratory parameters of the population in this study.

\begin{tabular}{|c|c|c|c|}
\hline \multicolumn{2}{|l|}{ Age (years) } & \multicolumn{2}{|l|}{$3-16$} \\
\hline \multicolumn{2}{|l|}{ Gender } & $n$ & $\%$ \\
\hline \multicolumn{2}{|l|}{ Male } & 40 & 57.1 \\
\hline \multicolumn{2}{|l|}{ Female } & 30 & 42.9 \\
\hline \multicolumn{2}{|c|}{ Parental geographic origin } & $n$ & $\%$ \\
\hline \multicolumn{2}{|c|}{ Angola } & 42 & 60.0 \\
\hline \multicolumn{2}{|c|}{ São Tomé and Príncipe Islands } & 8 & 11.4 \\
\hline \multicolumn{2}{|l|}{ Cape Verde } & 5 & 7.1 \\
\hline \multicolumn{2}{|c|}{ Guinea-Bissau } & 7 & 10.0 \\
\hline \multicolumn{2}{|c|}{ Guinea-Conakry } & 1 & 1.4 \\
\hline \multicolumn{2}{|l|}{ Nigeria } & 1 & 1.4 \\
\hline \multicolumn{2}{|c|}{ Double origin } & 6 & 8.6 \\
\hline \multicolumn{2}{|c|}{ Neurological status $(n=70)$} & $n$ & $\%$ \\
\hline \multicolumn{2}{|c|}{$\begin{array}{ll}\text { Stroke } & \text { Yes }\end{array}$} & 15 & 24.2 \\
\hline \multirow{3}{*}{$\begin{array}{r}\quad(n=62) \\
\text { SCI } \\
\quad(n=53)\end{array}$} & No & 47 & 75.8 \\
\hline & Yes & 9 & 16.9 \\
\hline & No & 44 & 83.0 \\
\hline $\mathrm{CV}$ & Yes & 24 & 38.7 \\
\hline \multirow{4}{*}{$\begin{array}{l}\quad(n=62) \\
\text { Stroke risk } \\
\quad(n=60)\end{array}$} & No & 38 & 61.3 \\
\hline & High $($ TAMMV $\geq 200 \mathrm{~cm} / \mathrm{s}$ ) & 25 & 41.7 \\
\hline & Moderate $(170 \mathrm{~cm} / \mathrm{s} \leq \mathrm{TAMMV} \leq 200 \mathrm{~cm} / \mathrm{s})$ & 6 & 10.0 \\
\hline & Low risk $($ TAMMV $<170 \mathrm{~cm} / \mathrm{s})$ & 29 & 48.3 \\
\hline \multicolumn{2}{|c|}{ Hematological parameters } & Median & IQR \\
\hline \multicolumn{2}{|l|}{ Hb S (\%) } & 79.9 & 14.5 \\
\hline \multicolumn{2}{|l|}{$\mathrm{Hb} F(\%)$} & 10.7 & 11.7 \\
\hline \multicolumn{2}{|l|}{$\mathrm{Hb}(\mathrm{g} / \mathrm{dL})$} & 8.0 & 1.3 \\
\hline \multicolumn{2}{|c|}{$\mathrm{RBC}\left(\times 10^{12} / \mathrm{L}\right)$} & 3.0 & 0.7 \\
\hline \multicolumn{2}{|l|}{ MCV (fL) } & 81.3 & 14.3 \\
\hline \multicolumn{2}{|l|}{$\mathrm{MCH}(\mathrm{pg})$} & 26.9 & 6.0 \\
\hline \multicolumn{2}{|c|}{ Reticulocytes (\%) } & 9.9 & 6.3 \\
\hline RDW (\%) & & 21.2 & 4.5 \\
\hline Platelets $(x$ & $\left.0^{9} / \mathrm{L}\right)$ & 404.1 & 167.7 \\
\hline WBC $\left(\times 10^{9}\right.$ & & 12.6 & 4.8 \\
\hline Neutrophils & $\left.\times 10^{9} / \mathrm{L}\right)$ & 5.6 & 2.65 \\
\hline Biochemical pa & rameters & Median & IQR \\
\hline LDH (U/L) & & 636.3 & 473.4 \\
\hline Total bilirub & $\mathrm{n}(\mathrm{mg} / \mathrm{dL})$ & 2.6 & 1.9 \\
\hline$H B B$ cluster ha & olotype & $n$ & $\%$ \\
\hline Bantu/Bantu & & 38 & 54.3 \\
\hline Senegal/Sen & gal & 11 & 15.7 \\
\hline Benin/Benin & & 3 & 4.3 \\
\hline Compound $\mathrm{h}$ & eterozygous & 17 & 24.2 \\
\hline Atypical & & 1 & 1.4 \\
\hline
\end{tabular}

A total of seventy-one genetic variants were identified of which twenty-eight (MAF $>0.05)$ were used in the association studies seven in VCAM1, five in NOS3, three in GOLGB1, one in PON1, one in ENPP1, one in HBA (- $\alpha^{3.7 \mathrm{~kb}}$-thal) and ten in ITGA4 (Appendix Table A.1). We were able to reconstruct 16 haplotypes, and used ten of them (frequency $>0.05$ ) for statistical analysis. Concerning the $H B B$ gene cluster haplotypes, only the more frequent genotypes, Bantu/Bantu and Senegal/Senegal, were used for statistical analysis (frequency $>0.05$ ).

\subsection{In silico analysis}

In silico analysis of the VCAM1 gene promoter variants was focused on those with MAF $>0.05$, except rs3917025 due to its occurrence in only one haplotype (Haplotype 3). The rs1041163_C, rs1409419_T and rs3917025_delCT VCAM1 alleles were classified (according to ClinVar and Ensembl's VEP and Mat Inspector) as intergenic variants with potential modifying impact, although with no major pathologic effects. Potential changes resulting from the presence of the rs1041163_C allele include (i) TFBS alteration for RXRF transcription factor, substituting it for a PRDF site, and (ii) loss of a FHXB TFBS. The presence of rs1409419_T would lead to a potential gain of binding sites, in particular, for EVI1, Oct1 and Barx2. Regarding rs3917025_delCT, a potential gain of a FAST1 (FoxH1) TFBS was indicated. 
Table 2

Association of biochemical and hematological parameters of SCA patients with stroke and stroke risk.

\begin{tabular}{|c|c|c|c|c|}
\hline \multirow[t]{3}{*}{ Parameter (units) } & \multirow[t]{3}{*}{$\mathrm{n}$} & \multicolumn{2}{|l|}{ Stroke } & \multirow[t]{3}{*}{$p$} \\
\hline & & Yes & No & \\
\hline & & Medians (IQR) & Medians (IQR) & \\
\hline $\mathrm{HbF}(\%)$ & 64 & $3.2(9.3)$ & $11.9(10.3)$ & 0.018 \\
\hline MCH (pg) & 70 & $21.2(20.8)$ & $27.4(6.0)$ & 0.005 \\
\hline \multirow[t]{3}{*}{ Parameter (units) } & \multirow[t]{3}{*}{$\mathrm{n}$} & \multicolumn{2}{|l|}{ Stroke risk } & \multirow[t]{3}{*}{$p$} \\
\hline & & High + moderate & Low & \\
\hline & & Medians (IQR) & Medians (IQR) & \\
\hline HbF (\%) & 64 & $8.5(10.2)$ & $12.1(10.7)$ & 0.043 \\
\hline Platelets $\left(\times 10^{9} / \mathrm{L}\right)$ & 61 & $442.0(156.4)$ & $363.2(124.2)$ & 0.017 \\
\hline Neutrophils $\left(\times 10^{9} / \mathrm{L}\right)$ & 61 & $6.3(2.5)$ & $4.9(2.6)$ & 0.009 \\
\hline LDH (U/L) & 65 & 761.5 (535.7) & $510.0(325.3)$ & $<0.001$ \\
\hline
\end{tabular}

Values indicated as medians (interquartile range in brackets); $\mathrm{n}$ - number of patients; HbF - hemoglobin F; MCH - mean corpuscular hemoglobin; LDH lactate dehydrogenase.

\subsection{Association of biochemical and hematological parameters with cerebral vasculopathy}

We observed significant associations of both stroke and stroke risk with several biochemical and hematological parameters (Table 2). Lower HbF percentages and MCH values were positively associated with stroke, while stroke risk was associated not only to lower $\mathrm{HbF}$ percentage but also to higher levels of coagulation, inflammation and hemolysis markers (Table 2).

\subsection{Association of genetic variants with biochemical and hematological parameters}

Genetic variants analyzed in our study, using the dominant genetic test model, showed association with both hematological and biochemical parameters, whether individually or as part of specific haplotypes (Table 3). VCAM1 rs1041163 (CC + TC), VCAM1_haplotype 3, ITGA4 rs113276800 (CA) and ITGA4 rs3770138 (TT + CT) showed an association with lower levels of $\mathrm{HbS}$. Conversely, higher $\mathrm{HbF}$ percentages were observed in association with rs1041163 (CC + TC), VCAM1_haplotype 3 and with Senegal/Senegal haplotype.

Namely, VCAM1 as well as ITGA4 variants, individually or within a haplotype context, were significantly associated with traditional biomarkers of disease severity, such as lower HbS percentage and higher $\mathrm{LDH}$ and total bilirubin values.

As for PON1 rs662, the AA and GA genotypes showed a positive association with high platelets counts characteristic of a pro-coagulant state. Regarding GOLGB1, no significant association was found between the presence of rs3742410_C and hematological or biochemical parameters. However, we found two other GOLGB1 SNPs while analyzing rs3732410_C - rs61746571_G and rs33988592_A, a synonymous and a missense variant, respectively. The rs61746571_G seems to be in linkage disequilibrium with variant rs3732410_C. On the other hand, rs33988592 AA and GA genotypes showed an association with lower values of inflammation markers (Table 3 ).
Table 3

Genetic variants association with the hematological and biochemical parameters.

\begin{tabular}{|c|c|c|c|c|c|}
\hline Gene & Variant & $\begin{array}{l}\text { Allele change or } \\
\text { haplotype }\end{array}$ & \multicolumn{2}{|c|}{$\begin{array}{l}\text { Parameter } \\
\text { (unit; nr. patients) }\end{array}$} & $p$ \\
\hline & & & \multicolumn{2}{|c|}{ HbS $(\% ; n=60)$} & \\
\hline & & & Var & No var & \\
\hline \multirow[t]{3}{*}{ VCAM1 } & rs1041163 & $\mathrm{T}>\mathrm{C}$ & 74.5 & 83.5 & 0.019 \\
\hline & Haplotype 3 & $\mathrm{C} / \mathrm{C} / \mathrm{CT} / \mathrm{T} / \mathrm{C} / \mathrm{C}$ & 73.7 & 81.4 & 0.034 \\
\hline & rs33988592 & $\mathrm{G}>\mathrm{A}$ & 11.3 & 13.4 & 0.030 \\
\hline \multirow[t]{4}{*}{ ITGA4 } & rs113276800 & $C>A$ & 69.0 & 80.4 & 0.012 \\
\hline & rs3770138 & $\mathrm{C}>\mathrm{T}$ & 66.1 & 80.7 & 0.003 \\
\hline & & & \multicolumn{2}{|c|}{$\mathrm{HbF}(\% ; n=64)$} & \\
\hline & & & Var & No var & \\
\hline \multirow[t]{2}{*}{ VCAM1 } & rs1041163 & $\mathrm{T}>\mathrm{C}$ & 13.0 & 7.0 & 0.014 \\
\hline & Haplotype 3 & $\mathrm{C} / \mathrm{C} / \mathrm{CT} / \mathrm{T} / \mathrm{C} / \mathrm{C}$ & 14.5 & 9.3 & 0.005 \\
\hline \multirow[t]{3}{*}{$H B B$} & Haplotype & Sen/Sen & 13.6 & 9.2 & 0.038 \\
\hline & & & \multicolumn{2}{|c|}{$\mathrm{Hb}(\mathrm{g} / \mathrm{dL} ; n=65)$} & \\
\hline & & & Var & No var & \\
\hline \multirow[t]{2}{*}{$H B B$} & Haplotype & Sen/Sen & 8.1 & 7.9 & 0.022 \\
\hline & & & \multicolumn{2}{|c|}{$\begin{array}{l}\mathrm{RBC}\left(\times 10^{12} / \mathrm{L}\right. \\
\mathrm{n}=64)\end{array}$} & \\
\hline \multirow[t]{2}{*}{$H B A$} & $-\alpha^{3.7 \mathrm{~kb}} \mathrm{del}$ & $\alpha \alpha>-\alpha^{3.7 \mathrm{~kb}}$ & 3.1 & 2.7 & 0.008 \\
\hline & & & \multicolumn{2}{|c|}{ MCV (fL; $n=69$ ) } & \\
\hline \multirow[t]{2}{*}{ NOS3 } & rs2070744 & $\mathrm{T}>\mathrm{C}$ & 87.3 & 79.9 & 0.024 \\
\hline & Haplotype IV & $\mathrm{T} / 4 \mathrm{a} / \mathrm{G}$ & 78.7 & 83.2 & 0.032 \\
\hline$H B A$ & $-\alpha^{3.7 \mathrm{~kb}} \mathrm{del}$ & $\alpha \alpha>-\alpha^{3.7 \mathrm{~kb}}$ & 75.7 & 85.7 & $<0.001$ \\
\hline \multirow[t]{3}{*}{$H B B$} & Haplotype & Sen/Sen & 90.1 & 80.6 & 0.039 \\
\hline & & & \multicolumn{2}{|c|}{ MCH (pg; $n=70)$} & \\
\hline & & & Var & No var & \\
\hline \multirow[t]{3}{*}{$H B A$} & $-\alpha^{3.7 \mathrm{~kb}} \mathrm{del}$ & $\alpha \alpha>-\alpha^{3.7 k b}$ & 25.1 & 29.0 & 0.001 \\
\hline & & & \multicolumn{2}{|c|}{$\mathrm{RDW}(n=70)$} & \\
\hline & & & Var & No var & \\
\hline \multirow[t]{2}{*}{ NOS3 } & rs2070744 & $\mathrm{T}>\mathrm{C}$ & 19.6 & 21.6 & 0.031 \\
\hline & Haplotype I & $\mathrm{C} / 4 \mathrm{a} / \mathrm{G}$ & 19.6 & 21.5 & 0.044 \\
\hline \multirow[t]{3}{*}{$H B A$} & $-\alpha^{3.7 \mathrm{~kb}}$ del & $\alpha \alpha>-\alpha^{3.7 \mathrm{~kb}}$ del & 20.8 & 21.2 & 0.021 \\
\hline & & & \multicolumn{2}{|c|}{$\begin{array}{l}\text { Platelets }\left(\times 10^{9} / \mathrm{L}\right. \\
n=61)\end{array}$} & \\
\hline & & & Var & No var & \\
\hline \multirow[t]{2}{*}{ PON1 } & rs662 & $\mathrm{G}>\mathrm{A}$ & 442.0 & 378.1 & 0.028 \\
\hline & & & $\begin{array}{l}\text { WBC } \\
n=6\end{array}$ & $9 / \mathrm{L}$ & \\
\hline GOLGB1 & rs33988592 & $\mathrm{G}>\mathrm{A}$ & 11.3 & 13.4 & 0.030 \\
\hline$H B B$ & Haplotype & Sen/Sen & 9.9 & 13.0 & 0.030 \\
\hline & & & $\begin{array}{l}\text { Neutr } \\
n=6\end{array}$ & $s\left(\times 10^{9} / \mathrm{I}\right.$ & \\
\hline & & & Var & No var & \\
\hline GOLGB1 & rs33988592 & $\mathrm{G}>\mathrm{A}$ & 5.0 & 6.2 & 0.013 \\
\hline$H B A$ & $-\alpha^{3.7 \mathrm{~kb}} \mathrm{del}$ & $\alpha \alpha>-\alpha^{3.7 \mathrm{~kb}}$ & 5.7 & 5.5 & 0.010 \\
\hline$H B B$ & Haplotype & Bantu/Bantu & 6.2 & 5.0 & 0.036 \\
\hline & Haplotype & Sen/Sen & 4.7 & 6.2 & 0.014 \\
\hline & & & LDH ( & $n=65)$ & \\
\hline & & & Var & No var & \\
\hline VCAM1 & rs1409419 & $C>T$ & 748.0 & 517.0 & $<0.001$ \\
\hline & Haplotype 7 & $\mathrm{~T} / \mathrm{C} / \mathrm{CT} / \mathrm{T} / \mathrm{T} / \mathrm{C}$ & 748.0 & 517.0 & $<0.001$ \\
\hline ITGA4 & Haplotype A & $\mathrm{G} / \mathrm{GA} / \mathrm{TT}$ & 611.5 & 1269.0 & 0.003 \\
\hline & & & $\begin{array}{l}\text { Biliru } \\
n=6\end{array}$ & $\mathrm{mg} / \mathrm{dL}$ & \\
\hline & & & Var & No var & \\
\hline VCAM1 & rs3783613 & $G>C$ & 3.2 & 2.4 & 0.026 \\
\hline
\end{tabular}

Var - presence of variant allele or haplotype; No var-absence of variant allele or haplotype; HbS - hemoglobin S; HbF - fetal hemoglobin; $\mathrm{Hb}$ - total hemoglobin; RBC - red blood cells; MCV - mean corpuscular volume; MCH mean corpuscular hemoglobin; RDW - red cell distribution width; WBC - white blood cells; LDH - lactate dehydrogenase; Sen - Senegal. 
Table 4

Genetic variants association with cerebral vasculopathy.

\begin{tabular}{|c|c|c|c|c|c|c|c|}
\hline \multirow[t]{2}{*}{ Gene } & \multirow[t]{2}{*}{ Variant } & \multicolumn{6}{|c|}{ Stroke $(n=62)$} \\
\hline & & Yes & No & & $p$ & OR & $95 \% \mathrm{CI}$ \\
\hline \multirow[t]{4}{*}{ VCAM1 } & rs1409419_TT + CT & 12 & 23 & & 0.041 & 4.17 & $1.04-16.73$ \\
\hline & rs1409419_CC & 3 & 24 & & & & \\
\hline & Haplotype 7 & 12 & 23 & & 0.041 & 4.17 & $1.04-16.73$ \\
\hline & Haplotype X & 3 & 24 & & & & \\
\hline \multirow[t]{4}{*}{ ITGA4 } & rs113276800_CA & 4 & 3 & & 0.025 & 7.62 & $1.39-41.65$ \\
\hline & rs113276800_CC & 7 & 40 & & & & \\
\hline & rs3770138_TT + CT & 4 & 4 & & 0.045 & 5.57 & $1.12-27.67$ \\
\hline & rs3770138_CC & 7 & 39 & & & & \\
\hline \multirow[t]{2}{*}{ Gene } & Variant & \multicolumn{6}{|l|}{$\mathrm{SCI}(n=53)$} \\
\hline & & Yes & No & & $p$ & OR & $95 \% \mathrm{CI}$ \\
\hline \multirow[t]{4}{*}{ NOS3 } & VNTR_4b & 6 & 42 & & 0.030 & 0.10 & $0.01-0.69$ \\
\hline & VNTR_4a + 4c & 3 & 2 & & & & \\
\hline & Haplotype V & 4 & 36 & & 0.031 & 0.18 & $0.04-0.81$ \\
\hline & Haplotype Y & 5 & 8 & & & & \\
\hline \multirow[t]{2}{*}{ Gene } & Variant & \multicolumn{6}{|l|}{$\mathrm{CV}(n=62)$} \\
\hline & & Yes & No & & $p$ & OR & $95 \% \mathrm{CI}$ \\
\hline \multirow[t]{2}{*}{ NOS3 } & Haplotype VII & 1 & 23 & & 0.006 & 0.08 & $0.01-0.69$ \\
\hline & Haplotype Z & 13 & 25 & & & & \\
\hline \multirow[t]{2}{*}{ Gene } & Variant & \multicolumn{6}{|l|}{ Stroke risk $(n=60)$} \\
\hline & & High + moderate & & Low & $p$ & OR & $95 \% \mathrm{CI}$ \\
\hline \multirow[t]{4}{*}{ VCAM1 } & rs1409419_TT + CT & 23 & & 11 & 0.009 & 4.71 & $1.57-14.13$ \\
\hline & rs1409419_CC & 8 & & 18 & & & \\
\hline & Haplotype 7 & 23 & & 11 & 0.009 & 4.71 & $1.57-14.13$ \\
\hline & Haplotype X & 8 & & 18 & & & \\
\hline \multirow[t]{2}{*}{ NOS3 } & Haplotype V & 18 & & 24 & 0.050 & 0.29 & $0.09-0.96$ \\
\hline & Haplotype Y & 13 & & 5 & & & \\
\hline \multirow[t]{2}{*}{ ENPP1 } & rs1044498_AA + CA & 14 & & 5 & 0.026 & 4.03 & $1.21-13.42$ \\
\hline & rs1044498_CC & 16 & & 23 & & & \\
\hline
\end{tabular}

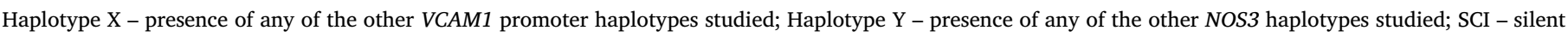
cerebral infarction; CV - cerebral vasculopathy; $95 \%$ CI - 95\% confidence interval.

\subsection{Association of genetic variants with cerebral vasculopathy and cerebral vasculopathy risk}

We found a significant association between the presence of several of the variants identified and CV/CV risk (Table 4). Namely, VCAM1 rs1409419 (TT + CT), haplotype 7, ITGA4 promoter rs113276800 (CA) and rs3770138 (TT + CT), showed a positive association with stroke. ITGA4 variant rs3770138 (TT + CT) was also positively associated with $\mathrm{CV}$ as a whole. Positive associations were also found between high TAMMV values and VCAM1 rs1409419 (TT + CT), VCAM1 promoter haplotype 7, and also with the ENPP1 rs1044498_A allele. On the other hand, NOS3 intron 4 VNTR_4b allele and haplotype V were negatively associated with SCI, while haplotype VII showed a negative association with CV overall.

\section{Discussion}

Our study aimed to assess demographic, clinical, biochemical, hematological, genotyping and imaging data to design a potential biomarker panel for CV prognosis in children with SCA. With this approach, we were able to identify statistically significant associations of biochemical, as well as hematological parameters, with genetic variants and CV.
In silico analysis of the VCAM1 rs1409419_T allele indicated particularly interesting potential TFBS gain, namely for EVI1, Oct1 and Barx2 transcription factors. EVI1 is of special note due to its complexity, multiple targets and modulation of several numerous processes, including cell migration, adhesion and proliferation [16]. It may cooperate with FOS transcription factor to limit cell adhesion while enhancing cell proliferation [16]. Conversely, Oct1 is a TF known to promote a transcriptional repression/silencing effect, which would potentially result in VCAM1 down-regulation. On the other hand, a Barx2 binding site gain, predicted as a result of the rs1409419_T presence, has been shown to promote murine muscle cell differentiation, by interacting with muscle regulatory factors, whereas its loss would lead to decreased adhesion properties [17]. Hence, it is reasonable to assume that a gain could result in increased adhesion properties. All the TFs which expression may be affected by the significantly associated variants are mainly involved in development and in different tissues following the proposed role of VCAM-1 [18]. It is important to emphasize that while we can address the potential effects of the individual VCAM1 variants' genotypes, the most important modifying role on disease manifestation would probably arise in the context of the haplotypes that encompass them. The one exception seems to be rs1409419_T given the overlapping findings observed for this variant and haplotype 7 , which includes it. 
We found that variants in the gene that encodes the VCAM-1 ligand, ITGA4, namely rs113276800_A and rs3770138_T were also positively associated with stroke. Furthermore, the latter was associated with CV globally. Given its role in WBC, reticulocyte and sickle erythrocyte adhesion to the activated SCA endothelium, this finding further underlines the role of cell-endothelium adhesion in the severe CV subphenotype. ITGA4 rs113276800 has been previously described in association with multiple sclerosis and, as in our case, no AA individuals were observed $[19,20]$. This variant is located in the ITGA4 promoter region near the AP-2 binding sites and the AA genotype may, therefore, cause a negative expression of the integrin $\alpha 4$ subunit [21]. We also found that the ITGA4 rs1375493, rs35723031 and rs10562650 variants behaved similarly in our group of patients, most of whom were heterozygous for the three of them simultaneously. Furthermore, the cooccurrence of minor alleles in haplotype A - which, to our knowledge, has not been previously described - was associated with lower LDH values and potentially to a protective effect against hemolysis.

NOS3 (or eNOS) is the major NO-producer enzyme in the cardiovascular system, playing a crucial role in vascular tone control, vascular remodeling and proliferation. Furthermore, in SCA, NO bioavailability plays a very important modulating role, primarily through scavenging by cell-free hemoglobin [22]. The rs2070744 variant, located at position -786 of the NOS3 gene $5^{\prime}$ flanking region, has been correlated with cardiovascular disease, namely its $C$ allele, although there is still debate about how it affects mRNA and protein levels. In our study, we did not find any association of this variant with CV or stroke risk, which may be in accordance with previous reports of no significant differences between CC and TT genotypes effect on NOS3 promoter activity [23]. The fact that the rs2070744_C has different distributions in different ethnic backgrounds [24] may also be responsible, to some extent, for these differences, since that allele is more frequent in Caucasians and our study population is of sub-Saharan origin. However, we observed a significant association between CC and TC genotypes of this variant and lower RDW, which has been discussed as a possible biomarker of lower cerebrovascular disease risk [25]. Lower RDW values (or reduced anisocytosis) would potentially act as a protective factor in consonance with what we observed for NOS3_haplotype V, which includes allele C, and CV protection. Although rs2070744 has been described in association with cardiovascular disease [26], its role in ischemic stroke has not been consensual. NOS3_haplotype V also includes intron 4 VNTR_4b allele, a variant that showed a protective effect against SCI. However, no relationship with any of the $\mathrm{CV}$ presentations studied here was observed. On the other hand, the NOS3 rs1799983_T allele, which leads to aspartate for glutamate substitution at eNOS position 298, has been previously reported to be related to deficient eNOS caveolar localization and deficient shear stress response leading to reduced enzymatic activity. This SNP has been found in some populations to be more prevalent in patients with coronary artery disease, ischemic stroke and arterial hypertension [27]. However, in our study population, we did not observe any relationship of rs1799983 TT or GT genotypes with CV, biochemical or hematological parameters.

Several studies have identified other candidate gene polymorphisms as potentially affecting the risk of CV. However, the results published so far have been conflicting. A GWAS study by Flanagan et al. [9], performed in a large cohort of mainly African American SCA pediatric patients, showed a decreased risk of clinically overt stroke in association with GOLGB1 rs3732410_G (Y1212C) and ENPP1 rs1044498_C (K173Q) mutations, whereas PON1 rs662_C (Q192R) was associated with increased risk of stroke [9]). In the same study, GOLGB1 Y1212C was associated with reduced TCD velocities and lower frequencies of SCIs. Conversely, reports from Martella et al. [7] and Belisário et al. [10] indicated a link between ENPP1 rs1044498_A and increased stroke risk as well as high TCD velocities. In our study, the ENPP1 rs1044498_A allele was found in $18 \%$ of patients compared to $68.33 \%$ of Martella et al. [7], 26.08\% of Belisário et al. [10] and 5.08\% of Flanagan et al. [9], while homozygosity for the GOLGB1 rs3732410_G allele was not found in our patients as in Flanagan's studies but contrary to $1.67 \%$ in Martella's report. Homozygotes for the PON1 rs662_C allele occurred in a frequency of $10,3 \%$ in our study, whereas Martella et al. [7] and Flanagan et al. [9] reported $45 \%$ and $13.7 \%$, respectively. Of the three variants, only ENPP1 rs1044498 AA and AC genotypes showed a positive association with stroke risk. Notably, rs1044498_A is the minor allele, in our study group, while the variant allele (C) is the most frequent, which is in line with the reference population (African Yoruba) and contrary to what is described for the other reference populations. This may reflect a negative selection for the less advantageous allele - rs1044498_A in these populations. As for the PON1 rs662_C variant, albeit no association with stroke or global CV was apparent, we observed a positive association with high platelet levels, indicating a potential impact on hemostasis and inflammation.

The only consensual modifiers of SCD disease severity, so far, have been the persistence of $\mathrm{HbF}$ beyond infancy and the presence of $-\alpha^{3.7 \mathrm{~kb}}$-thal deletion. The co-inheritance of $-\alpha^{3.7 \mathrm{~kb}}$-thal and homozygous $\mathrm{HbS}$ mutation has been associated with an overall ameliorating effect on anemia, particularly a protective effect against stroke in children [28]. We did not find any direct relationship between the presence of the $-\alpha^{3.7 \mathrm{~kb}}$-thal deletion and stroke, although we did find that patients with $\alpha$-thal showed a higher RBC count, lower MCV and $\mathrm{MCH}$, consistent with previous reports [29,30] of ameliorating anemia factors. Other authors have also reported an absence of association between $-\alpha^{3.7 \mathrm{~kb}}$-thal presence and stroke protection [31,32]. Despite the small sample size in our study, we cannot exclude that population heterogeneity or other specific population characteristics may contribute for the lack of association observed. Additionally, the unexpected association with increased neutrophil count might lower the above mentioned potentially favorable effect by indicating a proinflammatory role. The latter was also found in subjects with the Bantu haplotype while the Senegal haplotype seems to have the opposite effect, ameliorating inflammation and the hematological indices. Nevertheless, in our study, no $H B B$ cluster haplotypes were found to be associated with $\mathrm{CV}$.

\section{Conclusion}

Although the sample size in our study limits extrapolation to the general SCA pediatric population, our results seem to reinforce the importance of genetic modulators in the pathophysiology of cerebral vasculopathy and provide clues for the discovery of novel targets for SCA therapy. Our findings lead us to suggest that a comprehensive biomarker panel that includes biochemical, hematological, imaging as well as genetic data may be very important for CV prediction, and prevention. Even though the patients we studied are subjected to environmental, both physical and social, factors different from those to which the populations their parents originated from have been exposed, the genetic modifiers described in our study, namely VCAM1 haplotype 7 and rs1409419, may provide further tools for CV prevention in SCA. Functional studies are of the utmost importance, not only for confirmation purposes, but also to assess the mechanisms by which the phenotype modulation may occur, and the potential use of these variants as novel genetic biomarkers of disease prognosis.

\section{Author statement}

Due to the sensitive nature of the question asked in this study, survey respondents were assured raw data would remain confidential and would not be shared.

\section{CRediT authorship contribution statement}

Marisa Silva:Methodology, Software, Formal analysis, Investigation, Data curation, Writing - original draft.Sofia Vargas:Methodology, Software, Formal analysis, Investigation, 
Data curation.Andreia Coelho:Methodology, Software, Formal analysis, Investigation, Data curation.Emanuel Ferreira:Methodology, Software, Investigation.Joana Mendonça:Methodology, Software.Luís Vieira:Methodology, Software, Funding acquisition.Raquel Maia:Resources, Writing - review \& editing. Alexandra Dias:Resources, Writing - review \& editing.Teresa Ferreira:Resources, Writing - review \& editing.Anabela Morais:Resources.Isabel Mota Soares:Resources, Writing - review \& editing.João Lavinha:Funding acquisition, Writing - review \& editing.Rita Silva:Resources, Writing - review \& editing.Paula Kjöllerström:Resources, Writing - review \& editing.Paula Faustino:Project administration, Funding acquisition, Methodology, Supervision, Writing - review \& editing.

\section{Declaration of competing interest}

The authors have no competing interests.

\section{Acknowledgments}

The authors wish to thank the SCA patients and their parents for their participation in this study. We would also wish to thank Prof. Constança Coelho for her support in statistical analyses and insightful review of the manuscript.

\section{Financial support}

This work was partially funded by Fundação para a Ciência e a Tecnologia (FCT) grant PIC/IC/83084/2007, ISAMB, and INSA project 2012DGH720. Additionally, it is a result of the GenomePT project (POCI-01-0145-FEDER-022184), supported by COMPETE 2020 Operational Programme for Competitiveness and Internationalisation (POCI), Lisboa Portugal Regional Operational Programme (Lisboa2020), Algarve Portugal Regional Operational Programme (CRESC Algarve2020), under the PORTUGAL 2020 Partnership Agreement, through the European Regional Development Fund (ERDF), FCT.

\section{Appendix A. Supplementary data}

Supplementary data to this article can be found online at https:// doi.org/10.1016/j.bcmd.2020.102436.

\section{References}

1] D.J. Weatherall, The inherited diseases of hemoglobin are an emerging global health burden, Blood 115 (2010) 4331-4336, https://doi.org/10.1182/blood2010-01-251348.

[2] S.D. Grosse, I. Odame, H.K. Atrash, D.D. Amendah, F.B. Piel, T.N. Williams, Sickle cell disease in Africa: a neglected cause of early childhood mortality, Am. J. Prev. Med. 41 (2011) S398-S405, https://doi.org/10.1016/j.amepre.2011.09.013.

[3] F.B. Piel, S.I. Hay, S. Gupta, D.J. Weatherall, T.N. Williams, Global burden of sickle cell anaemia in children under five, 2010-2050: modelling based on demographics, excess mortality, and interventions, PLoS Med. 10 (2013), https://doi.org/10. 1371/journal.pmed.1001484.

[4] K. Ohene-Frempong, S.J. Weiner, L.A. Sleeper, S.T. Miller, S. Embury, J.W. Moohr, D.L. Wethers, C.H. Pegelow, F.M. Gill, Cerebrovascular accidents in sickle cell disease: rates and risk factors, Blood. 91 (1998) 288-294.

[5] I.D. Buchanan, A. James-Herry, I. Osunkwo, The other side of abnormal: a case series of low transcranial doppler velocities associated with stroke in children with sickle cell disease, J. Pediatr. Hematol. Oncol. 35 (2013) 543-546, https://doi.org/ 10.1097/MPH.0b013e318279caae.

[6] G. Buchanan, E. Vichinsky, L. Krishnamurti, S. Shenoy, Severe sickle cell diseasepathophysiology and therapy, Biol. Blood Marrow Transplant. 16 (2010) S64-S67, https://doi.org/10.1016/j.bbmt.2009.10.001.

[7] M. Martella, N. Quaglia, A.C. Frigo, G. Basso, R. Colombatti, L. Sainati, Association between a combination of single nucleotide polymorphisms and large vessel cerebral vasculopathy in African children with sickle cell disease, Blood Cells Mol. Dis. 61 (2016) 1-3, https://doi.org/10.1016/j.bcmd.2016.07.005.
[8] A. Coelho, A. Dias, A. Morais, B. Nunes, E. Ferreira, I. Picanço, P. Faustino, J. Lavinha, Genetic variation in CD36, HBA, NOS3 and VCAM1 is associated with chronic haemolysis level in sickle cell anaemia: a longitudinal study, Eur. J. Haematol. 92 (2014) 237-243, https://doi.org/10.1111/ejh.12226.

[9] J.M. Flanagan, V. Sheehan, H. Linder, T.A. Howard, Y.D. Wang, C.C. Hoppe, B. Aygun, R.J. Adams, G.A. Neale, R.E. Ware, Genetic mapping and exome sequencing identify 2 mutations associated with stroke protection in pediatric patients with sickle cell anemia, Blood 121 (2013) 3237-3245, https://doi.org/10. 1182/blood-2012-10-464156.

[10] A.R. Belisário, R.R. Sales, N.E. Toledo, C. Velloso-Rodrigues, C.M. Silva, M.B. Viana, Association between ENPP1 K173Q and stroke in a newborn cohort of 395 Brazilian children with sickle cell anemia, Blood 126 (2015) 1259-1260, https://doi.org/10. 1182/blood-2015-05-645176.

[11] S.H. Orkin, H.H. Kazazian, S.E. Antonarakis, S.C. Goff, C.D. Boehm, J.P. Sexton, et al., Linkage of $\beta$-thalassaemia mutations and $\beta$-globin gene polymorphisms with DNA polymorphism in human ß-globin gene cluster, Nature 296 (1982) 627-631.

[12] C. Dodé, R. Krishnamoorthy, J. Lamb, Rapid analysis of $\alpha 3.7$ thalassaemia and adaanti3.7 triplication by enzymatic amplification analysis, Br. J. Haematol. 83 (1993) 105-111.

[13] J.T. Robinson, H. Thorvaldsdóttir, W. Winckler, M. Guttman, E.S. Lander, G. Getz J.P. Mesirov, Integrative genome viewer, Nat. Biotechnol. 29 (2011) 24-26, https://doi.org/10.1038/nbt.1754.Integrative.

[14] M.F. Iademarco, J.J. McQuillan, G.D. Rosen, D.C. Dean, Characterization of the promoter for vascular cell adhesion molecule-1 (VCAM-1), J. Biol. Chem. 267 (1992) 16323-16329 http://www.ncbi.nlm.nih.gov/entrez/query.fcgi? cmd= Retrieve\&db $=$ PubMed\&dopt $=$ Citation\&list_uids $=1379595 \% 5$ Cnhttp: $/ /$ www.jbc. org/content/267/23/16323.abstract.

[15] G.D. Rosen, T.M. Birkenmeier, D.C. Dean, Characterization of the $\alpha 4$ integrin gene promoter, Proc. Natl. Acad. Sci. U. S. A. 88 (1991) 4094-4098, https://doi.org/10. 1073/pnas.88.10.4094.

[16] E.A. Bard-Chapeau, J. Jeyakani, C.H. Kok, J. Muller, B.Q. Chua, J. Gunaratne, a. Batagov, P. Jenjaroenpun, V.A. Kuznetsov, C.-L. Wei, R.J. D'Andrea, G. Bourque, N.A. Jenkins, N.G. Copeland, Ecotopic viral integration site 1 (EVI1) regulates multiple cellular processes important for cancer and is a synergistic partner for FOS protein in invasive tumors, Proc. Natl. Acad. Sci. 109 (2012) 2168-2173, https:// doi.org/10.1073/pnas.1119229109.

[17] R. Meech, K.N. Gonzalez, M. Barro, A. Gromova, L. Zhuang, J.A. Hulin, H.P. Makarenkova, Barx2 is expressed in satellite cells and is required for normal muscle growth and regeneration, Stem Cells 30 (2012) 253-265, https://doi.org/ 10.1002/stem.777.

[18] M.F. Iademarco, J.J. McQuillan, D.C. Dean, Vascular cell adhesion molecule 1: contrasting transcriptional control mechanisms in muscle and endothelium, Proc. Natl. Acad. Sci. U. S. A. 90 (1993) 3943-3947 http://www.pubmedcentral.nih.gov/ articlerender.fcgi? artid $=46422 \&$ tool $=$ pmcentrez\&rendertype $=$ abstract.

[19] G.A. Heymann, H. Kiesewetter, A. Salama, Frequencies of $\alpha 4$ A3061G variants and identification of three new variants of the human integrin $\alpha 4$-subunit, Mol. Immunol. 39 (2003) 855-860, https://doi.org/10.1016/S0161-5890(03)00009-9.

[20] V. Andreoli, R. Cittadella, P. Valentino, F. Condino, A. La Russa, M. Liguori, I. Manna, P. Spadafora, R. Nisticò, D. Pirritano, A. Clodomiro, A. Quattrone, The role of VLA4 polymorphisms in multiple sclerosis: an association study, $\mathrm{J}$. Neuroimmunol. 189 (2007) 125-128, https://doi.org/10.1016/j.jneuroim.2007. 06.015 .

[21] K. Hilger-Eversheim, M. Moser, H. Schorle, R. Buettner, Regulatory roles of AP-2 transcription factors in vertebrate development, apoptosis and cell-cycle control, Gene 260 (2000) 1-12, https://doi.org/10.1016/S0378-1119(00)00454-6.

[22] C.H.D.R. Reiter, X.U.W. Ang, J.O.S.E.E.T.A. Antos, N.E.I.L.H. Ogg, R.I.O.C.A. Iii, A. L.A.N.N.S. Chechter, M.A.R.K.T.G. Ladwin, FreeHb limits NO availability in SCD, Nat. Med. 8 (2002) 1383-1389. doi:https://doi.org/10.1038/nm799.

[23] A.S. Sim, J. Wang, D. Wilcken, Xing Li Wang, Mspl polymorphism in the promoter of the human endothelial constitutive NO synthase gene in Australian caucasian population, Mol. Genet. Metab. 65 (1998) 62, https://doi.org/10.1006/mgme. 1998.2741

[24] J.E. Tanus-Santos, M. Desai, D.A. Flockhart, Effects of ethnicity on the distribution of clinically relevant endothelial nitric oxide variants, Pharmacogenetics 11 (2001) 719-725, https://doi.org/10.1097/00008571-200111000-00011.

[25] N. Li, H. Zhou, Q. Tang, Red blood cell distribution width: a novel predictive in dicator for cardiovascular and cerebrovascular diseases, Dis. Markers 2017 (2017), https://doi.org/10.1155/2017/7089493.

[26] D. Colomba, G. Duro, S. Corrao, C. Argano, T. Di Chiara, D. Nuzzo, F. Pizzo, G. Parrinello, R. Scaglione, G. Licata, Endothelial nitric oxide synthase gene polymorphisms and cardiovascular damage in hypertensive subjects: an Italian casecontrol study, Immun. Ageing 5 (2008) 4, https://doi.org/10.1186/1742-4933-5-4

[27] I. Armenis, V. Kalotychou, R. Tzanetea, P. Kollia, Z. Kontogeorgiou, D. Anastasopoulou, M. Mantzourani, M. Samarkos, K. Pantos, K. Konstantopoulos, I. Rombos, Prognostic value of T786C and G894T eNOS polymorphisms in sickle cell disease, Nitric Oxide Biol. Chem. 62 (2017) 17-23, https://doi.org/10.1016/j. niox.2016.11.002.

[28] G.J. Kato, M.T. Gladwin, M.H. Steinberg, Deconstructing sickle cell disease: reappraisal of the role of hemolysis in the development of clinical subphenotypes, Blood Rev. 21 (2007) 37-47, https://doi.org/10.1016/j.blre.2006.07.001.

[29] M.B. Rumaney, V.J. Ngo Bitoungui, A.A. Vorster, R. Ramesar, A.P. Kengne, J. Ngogang, A. Wonkam, The co-inheritance of alpha-thalassemia and sickle cell anemia is associated with better hematological indices and lower consultations rate 
in Cameroonian patients and could improve their survival, PLoS One 9 (2014) e100516, , https://doi.org/10.1371/journal.pone.0100516.

[30] M.M. Aleluia, C.C. Da Guarda, R.P. Santiago, T.C.C. Fonseca, F.I. Neves, R.Q. De Souza, L.A. Farias, F.A. Pimenta, L.M. Fiuza, T.N. Pitanga, J.R.D. Ferreira, E.V. Adorno, B.A.V. Cerqueira, M. de S. Gonçalves, Association of classical markers and establishment of the dyslipidemic sub-phenotype of sickle cell anemia, Lipids Health Dis. 16 (2017) 1-9, https://doi.org/10.1186/s12944-017-0454-1.

[31] I.F. Domingos, D.A. Falcão, B.L. Hatzlhofer, A.F. Cunha, M.N. Santos, D.M. Albuquerque, K.Y. Fertrin, F.F. Costa, R.C. Azevedo, C.G. Machado,
A.S. Araújo, A.R. Lucena-Araujo, M.A. Bezerra, Influence of the $\beta$ s haplotype and $\alpha$ thalassemia on stroke development in a Brazilian population with sickle cell anaemia, Ann. Hematol. 93 (2014), https://doi.org/10.1007/s00277-014-2016-1.

[32] S.L. Saraf, R.E. Molokie, M. Nouraie, C.A. Sable, L. Luchtman-Jones, G.J. Ensing, A.D. Campbell, S.R. Rana, X.M. Niu, R.F. Machado, M.T. Gladwin, V.R. Gordeuk, Differences in the clinical and genotypic presentation of sickle cell disease around the world, Paediatr. Respir. Rev. 15 (2014) 4-12, https://doi.org/10.1016/j.prrv. 2013.11.003. 